\title{
Maximização do lucro na substituição de fontes proteicas na ração de juvenis de dourado
}

Profit maximization in the substitution of protein sources for juveniles of dourado

\author{
Fernando Yamamoto ${ }^{1}$; Daniel Yokoyama Sonoda ${ }^{2}$; José Eurico Possebon Cyrino ${ }^{3}$ \\ 1 Mestrando do Programa de Pós-graduação em Ciência Animal e Pastagens, Departamento de \\ Zootecnia - Avenida Pádua Dias, 11. São Dimas. CEP 13418-900. Piracicaba (SP), Brasil \\ 2 PECEGE - Doutor, Pesquisador - Rua Alexandre Herculano, 120. Ed. JK, Sala T4. Jardim Elite. CEP \\ 13418-445. Piracicaba (SP), Brasil \\ 3 ESALQ/USP - Professor Associado do Departamento de Zootecnia - Avenida Pádua Dias, 11. São \\ Dimas. CEP 13418-900. Piracicaba (SP), Brasil
}

\section{Resumo}

Peixes ictiófagos têm alta exigência dietética em proteína e, consequentemente, as dietas para estas espécies encontram-se entre as mais caras no mercado de alimentos para animais. O objetivo deste trabalho foi determinar o ponto de máximo lucro na substituição de farinha de peixe por farelo de soja das dietas para a produção de juvenis de dourado Salminus brasiliensis. Grupos de 12 juvenis da espécie $(8,97 \pm 0,33 \mathrm{~g})$ foram estocados em tanques de 500 $\mathrm{L}$ em circuito fechado de circulação de água em um delineamento experimental aleatório $(n=4)$, e alimentados até saciedade aparente em duas refeições diárias por 100 dias com rações extrudadas isoproteicas (39\% proteína digestível) e isoenergéticas (3900 kcal energia digestível), contendo níveis graduais de substituição de farinha de peixe [FP] por farinha de soja [FS] (100\% FP, 80\% FP, 60\% FP, 40\% FP, 20\% FP, 0\% FP). Os índices de desempenho dos peixes foram analisados pelo método de maximização de lucro, considerando-se os preços por kg de venda do dourado $(R \$ 20,00)$, do $F S(R \$ 1,14)$ e da $F P(R \$ 3,50)$. O percentual de farinha de peixe nas dietas que maximiza o lucro foi de $83,51 \%$.

Palavras-chave: farelo de soja, farinha de peixe, Salminus brasiliensis

\begin{abstract}
Carnivore fish have high dietary protein requirement, and consequently diets for these species are among the most expensive in the feed market. The objective of this study was to determine the point of maximum profit for the replacement of dietary fishmeal by soybean meal in the production of Salminus brasiliensis juveniles. Groups of 12 juveniles of the species (8.97 $\pm 0.33 \mathrm{~g}$ ) were stocked in 500-L tanks in a closed water circulation system. It was a randomized experimental design $(n=4)$, and being fed to apparent satiation in two daily meals per 100 days with isoproteic (39\% digestible protein) and isoenergetic (3900 kcal digestible energy), extruded feeds, containing gradual levels of substitution of fishmeal [FP] by soybean meal [FS] $(100 \%$ $\mathrm{FP}, 80 \% \mathrm{FP}, 60 \% \mathrm{FP}, 40 \% \mathrm{FP}, 20 \% \mathrm{FP}, 0 \% \mathrm{FP}$ ). The performance indexes of fish were analyzed by the profit maximization method, considering the sales prices per $\mathrm{kg}$ of dourado $(\mathrm{R} \$$ $20.00)$, of $F S(R \$ 1.14)$ and of $F P(R \$ 3.50)$. The percentage of dietary fishmeal diets that maximized profit was $83.51 \%$.
\end{abstract}

Keywords: soybean meal, fishmeal, Salminus brasiliensis

\section{Introdução}

As características de elevado teor proteico (50-70\% proteína bruta [PB]), perfil adequado de aminoácidos e ácidos graxos poliinsaturados essenciais, e alta palatabilidade a tornam a farinha de peixe [FP] a fonte proteica ideal em rações para organismos aquáticos (Anderson et al., 1995; Hardy e Tacon, 2002; Cheng et al.,

\footnotetext{
Autor correspondente: <dysonoda@usp.br>

Enviado: 10 nov. 2016

Aprovado: 06 fev. 2017
} 
2003). Entretanto, a sustentabilidade da produção de peixes, em particular espécies ictiófagas como o dourado (Salminus brasiliensis), está ameaçada por esta dependência da farinha de peixe [FP] como principal fonte protéica em função da instabilidade nos preços e disponibilidade. Desta forma, para manter as atuais taxas de crescimento da aquicultura de forma sustentável é necessário reduzir os conteúdos de FP nas dietas dos organismos aquáticos(Sanz et al., 2000; Cho e Bureau, 2001; Watanabe, 2002; Tacon e Forster, 2003).

O desenvolvimento e formulação de dietas baseadas em fontes proteicas alternativas é um dos atuais focos da pesquisa em nutrição de peixes. Ingrediente considerado candidato a substituir a FP nas rações devem ter preço competitivo, permitir alto desempenho por unidade de proteína consumida, não afetar negativamente na qualidade do produto final, ter baixo potencial poluente, ser disponível no mercado e de fácil transporte e manejo durante o processamento das rações (Hardy e Tacon, 2002).

O uso de fontes proteicas vegetais como alternativa à FP é defendido por vários pesquisadores como resultado doelevado volume de produção, preço relativamente baixo e a possibilidade do uso de resíduos da agroindústria na obtenção destes ingredientes (Papatryphon e Soares Jr, 2001; Fournier et al., 2004; Espe et al., 2007; Hansen et al., 2007; Hernandéz et al., 2007; Martínez-Llorens et al., 2007). Entretanto, esses ingredientes podem conter níveis consideráveis de fatores antinutricionais [FAN] (Francis et al., 2001) e, em geral, têm perfil de aminoácidos essenciais [AAE] inadequado. Apesar dos FAN serem frequentemente termolábeis, mesmo baixos níveis de inclusão nas dietas podem prejudicr a utilização dos nutrientes das dietas e o desempenho dos peixes.

Entre os subprodutos agrícolas, o farelo de soja [FS] é o ingrediente vegetal mais comumente utilizado na alimentação de organismos aquáticos. Embora não tenha um perfil balanceado de AAE (especialmente aminoácidos sulfurosos), é o considerado um substituto satisfatório para a FP por causa de seu baixo custo e alto valor nutricional (Cho e Bureau, 2001). O uso do FS como substituto parcial da FP tem sido testado em diversas espécies de peixe, substituindo com sucesso de 18 a $40 \%$ da FP dependendo da espécie (Chong et al., 2003; Catacutan e Pagador, 2004; Zhou et al., 2005; Biswas et al., 2007; Hernandéz et al., 2007; Pham et al., 2007). No entanto, trabalhos avaliando sua economicidade são, no mínimo, escassos.

O dourado apresenta características comerciais e zootécnicas que o qualificam como espécie potencial para a piscicultura brasileira: rápido desenvolvimento inicial, 
carne de excelente qualidade, demanda pelo mercado consequente preço elevado (Della Flora et al., 2010; Weingartner e Zaniboni-Filho, 2010). Durante a fase adulta possui hábito alimentar ictiófago ou piscívoro por excelência, o que condiciona alta exigência dietética em proteína e consequente alta dependência de fontes proteicas de origem animal de alto custo, como a FP, de modos que as dietas para estas espécies se encontram entre as mais caras no mercado de alimentos para animais. $O$ objetivo deste trabalho foi então determinar o ponto de máximo lucro na substituição de farinha de peixe por farelo de soja na produção de juvenis de dourado.

\section{Material e Métodos}

Grupos de juvenis de dourado $(8,97 \pm 0,33 \mathrm{~g} ; \mathrm{n}=12)$ foram estocados em tanques de $500 \mathrm{~L}$ em circuito fechado de circulação de água, em um delineamento experimental totalmente aleatorizado $(n=4)$, e alimentados em duas refeições diárias por 100 dias com dietas isoproteicas (39\% proteína digestível) e isoenergéticas (3.900 kcal energia digestível) contendo níveis graduais de substituição de FS por FP 100\% FP, $80 \% \mathrm{FP}, 60 \% \mathrm{FP}, 40 \% \mathrm{FP}, 20 \% \mathrm{FP}, 0 \% \mathrm{FP})$. As rações foram formuladas segundo Borghesi et al. (2011) (Tabela 1), extrudadas e secas $\left(55^{\circ} \mathrm{C} ; 24 \mathrm{~h}\right.$ ) e armazenadas em câmara frigorífica até o momento do uso.

Tabela 1. Variação na composição da formulação com a introdução de farinha de peixe $[\mathrm{FP}]$ da ração para juvenis de Dourado

\begin{tabular}{|c|c|c|c|c|c|c|}
\hline Ingredientes & 100 & 80 & 60 & 40 & 20 & 0 \\
\hline & & & & & & \\
\hline Farinha de peixe & 59,80 & 47,84 & 35,88 & 23,82 & 13,11 & 0,00 \\
\hline Farinha de soja & 0,00 & 16,90 & 34,00 & 51,46 & 68,03 & 89,13 \\
\hline Farinha de trigo & 19,58 & 19,58 & 18,50 & 17,00 & 10,60 & 1,50 \\
\hline Amido & 13,00 & 8,06 & 4,00 & 0,00 & 0,00 & 0,00 \\
\hline le soja & 6,60 & 5,33 & 4,00 & 2,73 & 1,46 & 0,00 \\
\hline Óleo de p & 0,00 & 1,27 & 2,54 & 3,81 & 5,48 & 7,94 \\
\hline DL-Metionina & 0,00 & 0,00 & 0,06 & 0,16 & 0,30 & 0,41 \\
\hline Premix & 1,00 & 1,00 & 1,00 & 1,00 & 1,00 & 1,00 \\
\hline hidroxitolueno butilado [BHT] & 0,02 & 0,02 & 0,02 & 0,02 & 0,02 & 0,02 \\
\hline Nutrientes & \multicolumn{6}{|c|}{ Composição calculada das dietas } \\
\hline Proteína digestível (\%) & 38,99 & 38,98 & 38,97 & 39,03 & 38,95 & 39,02 \\
\hline Energia digestível (kcal) & 3927 & 3937 & 3935 & 3937 & 3920 & 3924 \\
\hline Fibra bruta (\%) & 1,08 & 2,00 & 2,88 & 3,76 & 4,31 & 4,97 \\
\hline Lipidio (\%) & 13,39 & 12,56 & 11,62 & 10,73 & 10,15 & 9,92 \\
\hline Lisina (\%) & 2,15 & 2,24 & 2,33 & 2,42 & 2,50 & 2,62 \\
\hline Metionina (\%) & 1,08 & 0,97 & 0,91 & 0,90 & 0,92 & 0,90 \\
\hline
\end{tabular}

Fonte: Dados originais da pesquisa

O método utilizado para a análise foi o da maximização de lucro, adaptado de Varian (1990) e Griffiths et al. (1993). A função lucro foi definida como a diferença 
entre a receita obtida com a venda do produto e os custos de produção do sistema para cada tratamento, conforme eq.(1):

$$
L_{i j}=R_{i j}-C_{i j}
$$

onde, $L_{i j}$ : função lucro; $R_{i j}$ : receita obtida com a venda do produto; $C_{i j}$ : custos de produção do sistema; i: introdução gradual da farinha de peixe na composição da ração; j: número de repetições.

A receita foi igual ao preço de venda do peixe multiplicado por sua respectiva quantidade produzida, de acordo com eq. (2):

$$
R_{i j}=P \cdot Y_{i j}
$$

onde, $\mathrm{R}_{\mathrm{ij}}$ é a receita obtida com a venda do produto; $(\mathrm{P})^{1}$ : preço de venda do peixe; $\mathrm{Y}_{\mathrm{ij}}$ : é a quantidade produzida.

O custo considerado foi igual ao somatório dos preços de cada componente da ração multiplicado por suas respectivas quantidades consumidas, conforme eq. (3):

$$
C_{i j}=\left(W_{i j 1} \cdot X_{i j 1}+W_{i j 2} \cdot X_{i j 2}+\cdots+W_{i j n} \cdot X_{i j n}\right)
$$

onde, $C_{i j:}$ custo; $W_{i j}$ : preço do componente da ração; $X_{i j}$ : quantidade de ração consumida.

Desta forma, pode-se reescrever a função lucro $\left(L_{i j}\right)$ de acordo com a eq. (4):

$$
L_{i j}=P \cdot Y_{i j}-\left[\left(W_{i j 1} \cdot X_{i j 1}+W_{i j 2} \cdot X_{i j 2}+\cdots+W_{i j n} \cdot X_{i j n}\right)\right]
$$

Estimou-se uma função lucro em função do percentual de farinha de peixe introduzida na ração, conforme eq. (5):

$$
L_{i j}=f(i)
$$

A forma funcional utilizada para o modelo foi a quadrática, estimada através de método de mínimos quadrados ordinários (Gujarati, 2000; Griliches e Intriligator,

${ }^{1} \mathrm{P} \in \mathrm{R}_{+} \rightarrow \mathrm{P}$ é uma variável exógena 
1990). Para maximizar a função lucro, derivou-se esta função em relação ao percentual de farinha de peixe introduzida na ração, obteve-se a eq. (6):

$$
\frac{\partial L}{\partial i}=0
$$

Igualando-se a derivada a zero, obteve-se percentual de farinha de peixe na ração que maximiza o lucro.

\section{Resultados e Discussões}

Apesar das rações formuladas serem isoproteicas e isoenergéticas, as conversões alimentares dos peixes tenderam a melhorar a medida que o percentual de farinha de peixe aumentou na ração (Figura 1). O crescimento dos peixes está diretamente relacionado ao respectivo consumo de ração. As rações com concentrações a partir de $40 \%$ de FP foram consumidas em maiores quantidades em relação às demais (Figura 2). A variação no consumo entre os níveis pode estar associada à palatabilidade das rações para a espécie, em que rações com maiores níveis de farinha de peixe seriam melhor aceitas que rações com maiores concentrações de farelo de soja. Eventualmente, o maior uso de flavorizantes como óleo de peixe poderia melhorar o consumo de rações com menores concentrações de farinha de peixe (Sampaio-Oliveira e Cyrino, 2004).

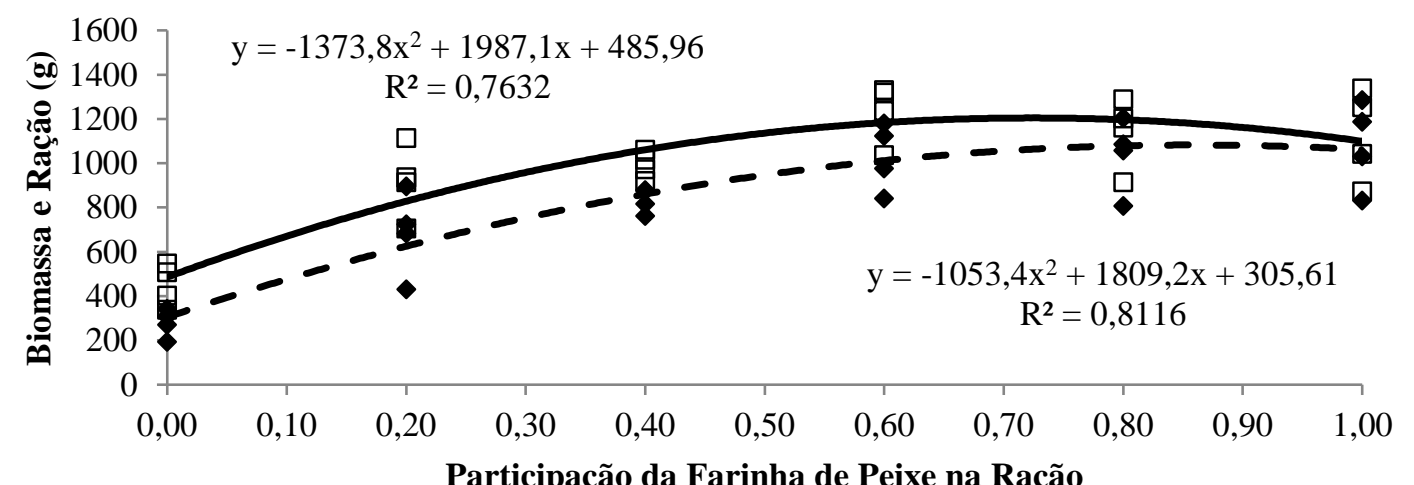

- Biomassa $\quad$ Ração - Polinômio (Biomassa) — Polinômio (Ração)

Figura 1. Curvas de produção de biomassa e consumo de ração em função da participação de farinha de peixe na ração

Fonte: Resultados originais da pesquisa 


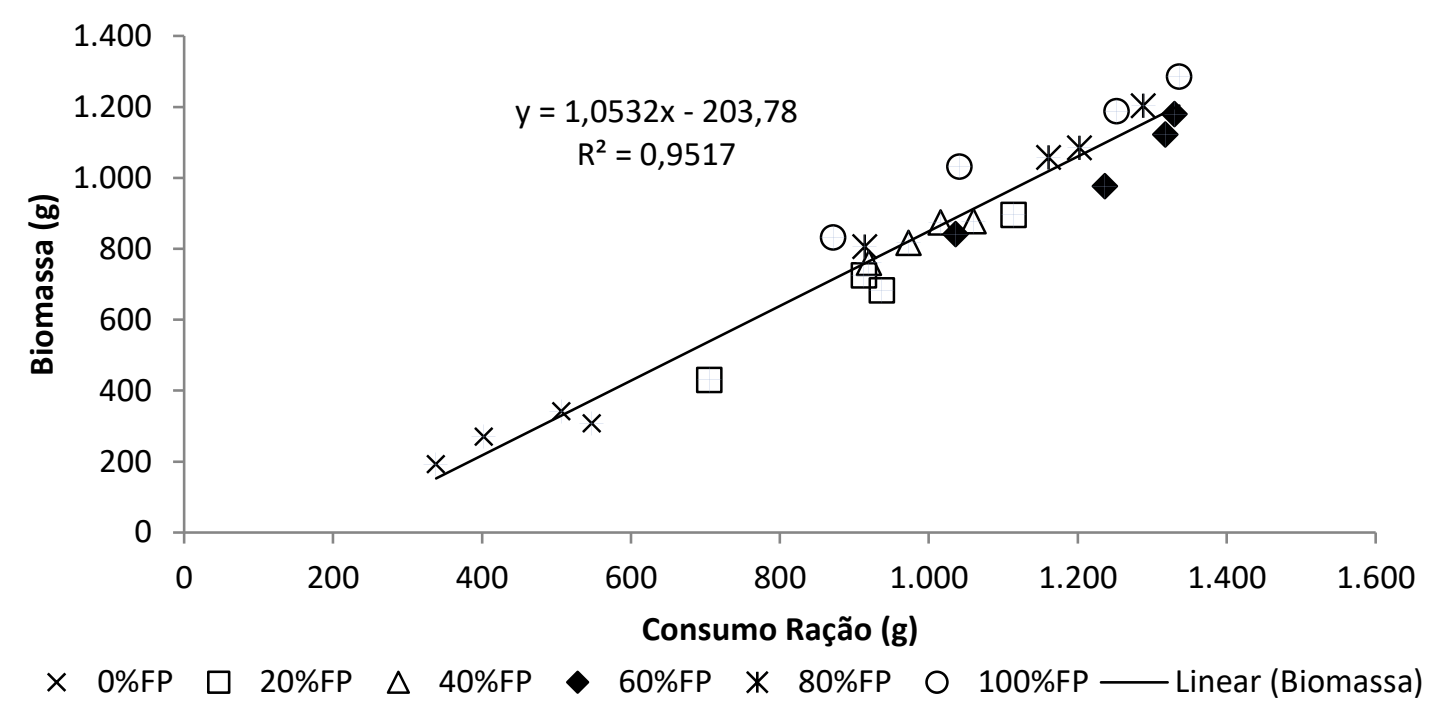

Figura 2. Curva de produção de biomassa em função do consumo de ração Fonte: Resultados originais da pesquisa

Para evitar distorções na análise, o custo dos demais componentes desta ração foi desprezado. Portanto, os preços considerados para a análise foram em reais $[R \$]$ de 2013 (Tabela 2).

Tabela 2. Preço dos principais componentes da ração (insumos) e do Dourado (produto) em 2013

\begin{tabular}{lc}
\hline \multicolumn{1}{c}{ Item } & Preço \\
\hline & 3,50 \\
Farinha de Peixe & 1,14 \\
Farelo de Soja & 20,00 \\
Dourado &
\end{tabular}

Fonte: Resultados originais da pesquisa

A partir dos preços e dos parâmetros de ganho de biomassa e consumo de ração foi calculada a receita, os custos com ração e o lucro ${ }^{2}$ [L] do experimento. Para o cálculo do custo de ração foram considerados apenas os valores dos componentes avaliados no experimento: farinha de peixe e farelo de soja. Por se tratar de uma ração experimental, os preços dos outros componentes [OC] utilizados para fechar a sua formulação não representam os preços dos componentes utilizados nas rações comerciais e poderiam distorcer os resultados da análise (Tabela 3).

\footnotetext{
2 Apesar de Margem Bruta (MB) ser a definição mais adequada para esta sitação, optou-se por manter o termo lucro [L] para mater a coerência com a metodologia adotada
} 
Tabela 3. Dados de farelo de soja [FS], participação da farinha de peixe na ração [i] e outros componentes [OC] utilizados para análise de regressão

\begin{tabular}{cccccccc}
\hline FS & i & OC & Biomassa & Ração & Receita & Custo & Lucro \\
\hline------------ & \\
89,13 & 0,00 & 10,87 & 269,90 & 402,37 & 5,40 & 0,41 & 4,99 \\
89,13 & 0,00 & 10,87 & 341,70 & 506,81 & 6,83 & 0,51 & 6,32 \\
89,13 & 0,00 & 10,87 & 192,40 & 338,38 & 3,85 & 0,34 & 3,50 \\
89,13 & 0,00 & 10,87 & 307,60 & 547,19 & 6,15 & 0,56 & 5,60 \\
68,03 & 13,11 & 18,86 & 724,50 & 912,90 & 14,49 & 1,13 & 13,36 \\
68,03 & 13,11 & 18,86 & 682,20 & 936,88 & 13,64 & 1,16 & 12,49 \\
68,03 & 13,11 & 18,86 & 429,70 & 705,39 & 8,59 & 0,87 & 7,72 \\
68,03 & 13,11 & 18,86 & 895,10 & 1113,99 & 17,90 & 1,38 & 16,53 \\
51,46 & 23,82 & 24,72 & 874,50 & 1015,75 & 17,49 & 1,44 & 16,05 \\
51,46 & 23,82 & 24,72 & 760,40 & 919,78 & 15,21 & 1,31 & 13,90 \\
51,46 & 23,82 & 24,72 & 816,30 & 972,83 & 16,33 & 1,38 & 14,94 \\
51,46 & 23,82 & 24,72 & 877,10 & 1059,98 & 17,54 & 1,51 & 16,04 \\
34,00 & 35,88 & 30,12 & 840,60 & 1035,91 & 16,81 & 1,70 & 15,11 \\
34,00 & 35,88 & 30,12 & 1122,20 & 1317,57 & 22,44 & 2,17 & 20,28 \\
34,00 & 35,88 & 30,12 & 1179,20 & 1330,18 & 23,58 & 2,19 & 21,40 \\
34,00 & 35,88 & 30,12 & 976,60 & 1236,35 & 19,53 & 2,03 & 17,50 \\
16,90 & 47,84 & 35,26 & 1084,50 & 1202,51 & 21,69 & 2,25 & 19,44 \\
16,90 & 47,84 & 35,26 & 1056,80 & 1161,04 & 21,14 & 2,17 & 18,97 \\
16,90 & 47,84 & 35,26 & 806,40 & 913,85 & 16,13 & 1,71 & 14,42 \\
16,90 & 47,84 & 35,26 & 1202,90 & 1288,24 & 24,06 & 2,41 & 21,65 \\
0,00 & 59,80 & 40,20 & 1187,20 & 1252,13 & 23,74 & 2,62 & 21,12 \\
0,00 & 59,80 & 40,20 & 831,20 & 871,56 & 16,62 & 1,82 & 14,80 \\
0,00 & 59,80 & 40,20 & 1285,30 & 1336,38 & 25,71 & 2,80 & 22,91 \\
0,00 & 59,80 & 40,20 & 1030,90 & 1040,93 & 20,62 & 2,18 & 18,44 \\
\hline
\end{tabular}

Fonte: Resultados originais da pesquisa

A partir dos dados de i e Lucro (Tabela 3) e que estão ilustrados na figura 3, a forma funcional foi obtida através da regressão, conforme eq. (7).

$$
L_{i j}=5,638443+33,00448 \cdot i-19,7605 \cdot i^{2}
$$

Os testes estatísticos indicam que os parâmetros (intercepto, i e $\mathrm{i}^{2}$ ) e o teste $\mathrm{F}$ são significativos à $1 \%$ e o $\mathrm{R}^{2}=0,799$ o que indica um bom ajuste da regressão aos dados. Derivando-se a função de lucro em relação ao percentual de farinha de peixe introduzida na ração, obtém-se a eq. (8), ilustrada na Figura 3:

$$
\begin{gathered}
\frac{\partial L}{\partial i}=33,00448-39,521 \cdot i=0 \\
i^{*}=83,51
\end{gathered}
$$




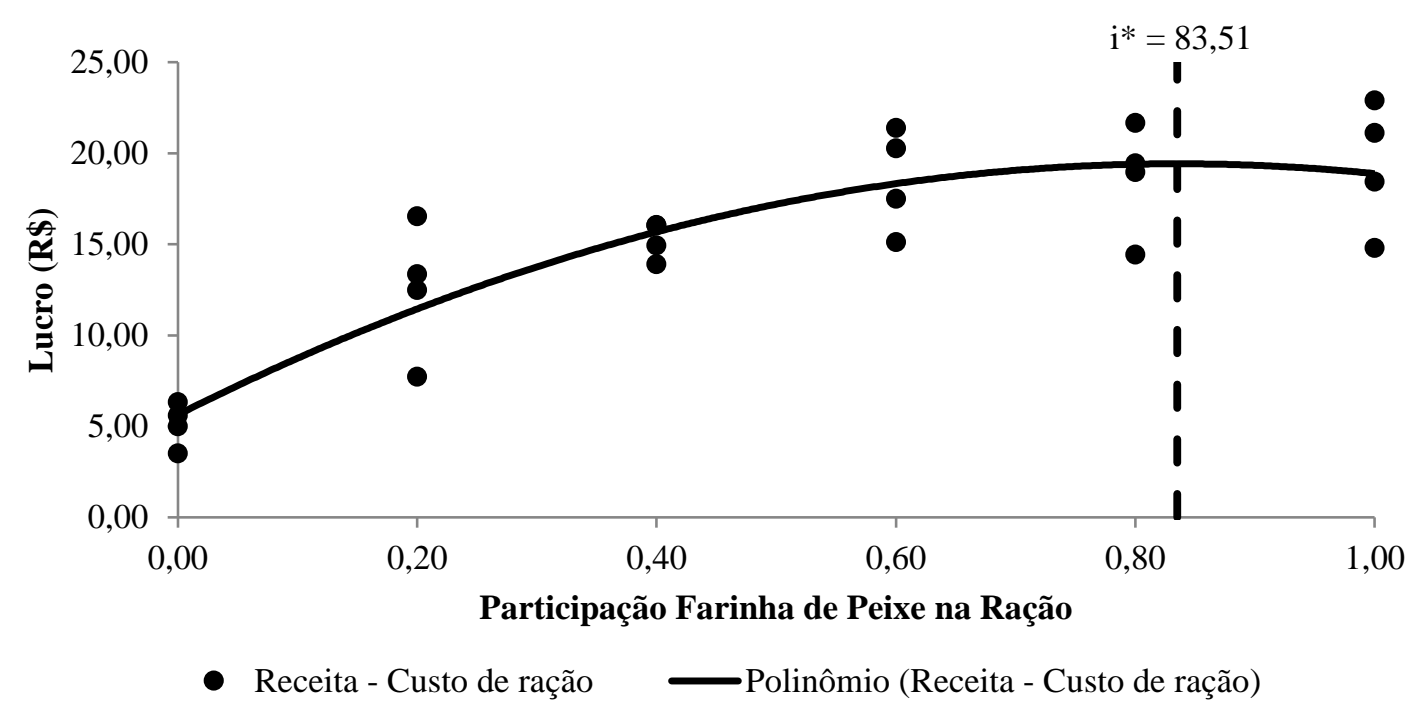

Figura 3. Lucro em função da participação de farinha de peixe na ração para juvenis de Dourado

Fonte: Resultados originais da pesquisa

A inclusão de $83,51 \%$ de farinha de peixe nas dietas maximizou o lucro na produção de juvenis de dourado. Embora o preço do farelo de soja seja um terço do preço da farinha de peixe, a redução do consumo da ração resultante da inclusão de farelo de soja na dieta causou redução na taxa de crescimento, ou seja, produziu menor biomassa, fator determinante para a redução do lucro da produção em piscicultura.

\section{Conclusão}

A inclusão de farelo de soja na dieta aumenta o lucro da produção de juvenis de dourado, sendo que o percentual de farinha de peixe na dieta que maximiza o lucro é de $83,51 \%$.

\section{Referências}

Anderson, J.S.; Lall, S.P.; Anderson, D.M.; McNiven, M.A. 1995. Availability of amino acids from various fish meals fed to Atlantic salmon (Salmo salar). Aquaculture 138: 291-301.

Biswas, A.K.; Kaku, H.; Ji, S.C.; Seoka, M.; Takii, K. 2007. Use of soybean meal and phytase for partial replacement of fish meal in the diet of red sea bream, Pagrus major. Aquaculture 267: 284-291.

Borghesi, R.; Dairiki, J.K.; Cyrino, J.E.P. 2009. Apparent digestibility coefficients of selected feed ingredients for dourado Salminus brasiliensis. Aquaculture Nutrition 15: 453-458. 
Catacutan, M.R.; Pagador, G. E. 2004. Partial replacement of fishmeal by defatted soybean meal in formulated diets for the mangrove red snapper, Lutjanus argentimaculatus (Forsskal 1775). Aquaculture Research 35: 299-306.

Cheng, Z.J.; Hardy, R.W.; Usry, J.L. 2003. Effects of lysine supplementation in plant protein-based diets on the performance of rainbow trout (Oncorhynchus mykiss) and apparent digestibility coefficients of nutrients. Aquaculture 215: 255-265.

Cho, C.Y.; Bureau, D.P. 2001. A review of diet formulation strategies and feeding systems to reduce excretory and feed wastes in aquaculture. Aquaculture Research 32: 349-360.

Chong, A.; Hashim, R.; bin Ali, A. 2003. Assessment of soybean meal in diets for discus (Symphysodon aequifasciata HECKEL) farming through a fishmeal replacement study. Aquaculture Research 34: 913-922.

Della Flora, M.A.; Maschke, F.; Ferreira C.C.; Pedron, F.A. 2010. Biologia e cultivo do Dourado (Salminus brasiliensis). Acta Veterinaria Brasilica 4: 7-14.

Espe, M.; Lemme, A.; Petri, A.; El-Mowafi, A. 2007. Assessment of lysine requirement for maximal protein accretion in Atlantic salmon using plant protein diets. Aquaculture 263: 168-178.

Fournier, V.; Huelvan, C.; Desbruyeres, E. 2004. Incorporation of a mixture of plant feedstuffs as substitute for fish meal in diets of juvenile turbot (Psetta maxima). Aquaculture 236: 451-465.

Francis, G.; Makkar, H. P. S.; Becker, K. 2001. Antinutritional factors present in plantderived alternate fish feed ingredients and their effects in fish. Aquaculture 199: 197227.

Griffiths, W.E.; Hill, R.C.; Judge, G.G. 1993. Learning and practicing econometrics. John Wiley \& Sons. New York, New York, USA.

Griliches, Z.; Intriligator, M.D. 1990. Handbook of econometrics. 2. ed. Elsevier Science Publisher, Amsterdam, Netherlands.

Gujarati, D.N. 2000. Econometria Básica. 3ed. Makron Books, São Paulo, São Paulo, Brasil.

Hansen, A.-C.; Karlsen, Ø., Rosenlund, G., Rimbach, M., \& Hemre, G.-I. 2007. Dietary plant protein utilization in Atlantic cod, Gadus morhua L. Aquaculture Nutrition 13: 200215.

Hardy, R.W.; Tacon, A.G.J. 2002. Fish meal: historical uses, production trends and future outlook for sustainable supplies. p. 311-325. In: Stickney, R.R.; McVey, J.P. Responsible marine aquaculture. CAB International, Cambridge, United Kingdom.

Hernandéz, M.D.; Martínez, F.J.; Jover, M.; García García, B. 2007. Effects of partial replacement of fish meal by soybean meal in sharpsnout seabream (Diplodus puntazzo) diet. Aquaculture 263: 159-167. 
Martínez-Llorens, S.; Moñino, A.V.; Vidal, A.T.; Salvador, V.J.M.; Torres, M.P.; Cerdá, M.J. 2007. Soybean meal as a protein source in gilthead sea bream (Sparus aurata L.) diets: effects on growth and nutrient utilization. Aquaculture Research 38: 82-90.

Papatryphon, E.; Soares Jr, J.H. 2001. Optimizing the levels of feeding stimulants for use in high-fish meal and plant feedstuff-based diets for striped bass, Morone saxatilis. Aquaculture 202: 279-288.

Pham, M.A.; Lee, K.J.; Lim, S.J.; Park, K.H. 2007. Evaluation of cottonseed and soybean meal as partial replacement for fishmeal in diets for juvenile Japanese flounder Paralichthys olivaceus. Fisheries Science 73: 760-769.

Sampaio-Oliveira, A.M.B.M.; Cyrino, J.E.P. 2004. Attractants in plant protein-based diets for the carnivorous largemouth bass Micropterus salmoides. Scientia Agricola $61(3) 326-331$.

Sanz, A.; Gallego, M.G.; De la Higuera, M. 2000. Protein nutrition in fish: protein:energy ratio and alternative protein sources to fish meal. Journal of Physiology and Biochemistry 56: 275-282.

Tacon, A.G.J.; Forster, I.P. 2003. Aquafeeds and the environment: policy implications. Aquaculture 226: 181-189.

Varian, H.R.1990. Microeconomia: princípios básicos. 4ed. Campus, Rio de Janeiro, Rio de Janeiro, Brasil.

Watanabe, T. 2002. Strategies for further development of aquatic feeds. Fisheries Science 68: 242-252.

Weingartner, M.; Zaniboni-Filho, E. 2005. Dourado. p. 257-286. In: Baldisserotto, B.; Gomes, L.C. Espécies nativas para piscicultura no Brasil. UFSM, Santa Maria, Rio Grande do Sul, Brasil.

Zhou, Q.-C.; Mai, K.-S. ; Tan, B.-P. ; Liu, Y.-J. 2005. Partial replacement of fish meal by soybean meal in diets for juvenile cobia (Rachycentron canadum). Aquaculture Nutrition 11: 175-182. 